\title{
DESIGN AND ANTICANCER ACTIVITY PREDICTION OF DIHYROPYRIMIDINONE BASED NOVEL INHIBITORS OF P53-MDM2 INTERACTION
}

\author{
SURENDRA KUMAR NAYAK ${ }^{1 *}$, GOPAL LAL KHATIK ${ }^{1}$, RAKESH NARANG ${ }^{1}$, HARISH KUMAR CHOPRA ${ }^{2}$ \\ ${ }^{1}$ Department of Pharmaceutical Chemistry, School of Pharmaceutical Sciences, Lovely Professional University, Jalandhar-Delhi, G. T. Road \\ (NH-1), Phagwara, Punjab -144 411, India. ${ }^{2}$ Department of Chemistry, Sant Longowal Institute of Engineering and Technology (Deemed \\ University), Longowal, Sangrur - 148 106, India. Email: surendra_niper@yahoo.com
}

Received: 14 July 2017, Revised and Accepted: 25 July 2017

ABSTRACT

Objective: P53 protein is well known for its role in cell cycle regulation and induction of apoptosis. This protein is degraded by MDM2 mediated proteolysis. Inhibition of interaction between p53 and MDM2 has been recognized as a most potential and selective target for development of novel anticancer agents. Recently, several molecules entered in the clinical trial study for the treatment of various types of cancers are based on inhibition of interaction between p53-MDM2. Therefore, in this study, a novel dihydropyridine based molecules were designed as p53-MDM2 inhibitor, and their anticancer activity (including reference) was determined in comparison with most active anticancer agent and inactive anticancer agents in National Cancer Institute database using "Cancer IN" server.

Methods: In this work, a novel dihydropyrimidinone based lead (L11) on the basis of molecular docking study, predicted IC ${ }_{50}$, anticancer activity, and toxicity profile were designed. Lead L11 was obtained after sequential isosteric replacement of functional groups for optimization in compound L0.

Results: The docking scores of L3-L11 found to be in range of 21-25 close to docking score 25 of SAR405838 and better than nutlin-3a. MDM2 binding affinity values (37-78 Kcal/mol) of all ligands were also found to better than that of nutlin-3a (37 Kcal/mol). Surprisingly, MDM2 binding affinity of L11 (78 Kcal/mol) found to be equal to that of SAR405838 and 2-fold greater than nutlin-3a.

Conclusion: These data indicating that L11 as a potential lead from dihydropyrimidinones for inhibition of p53-MDM2 interaction.

Keywords: Anticancer, Dihydropyrimidinone, MDM2, p53, Toxicity.

(C) 2017 The Authors. Published by Innovare Academic Sciences Pvt Ltd. This is an open access article under the CC BY license (http://creativecommons. org/licenses/by/4. 0/) DOI: http://dx.doi.org/10.22159/ajpcr.2017.v10s3.21346

\section{INTRODUCTION}

Most of the anticancer drugs act through direct or indirect induction of tumor suppressor protein $\mathrm{p} 53$. When level of functionally active p53 rises in tumor cells, then it destroy them through apoptosis. In several cancers, the level of functional active p53 is suppressed due to its MDM2 mediated proteolytic degradation [1]. The interaction between p53 and MDM2 is a key point for the development of various types of cancers. P53 binds in a deep hydrophobic cavity on MDM2 surface through its three hydrophobic amino acid residues (Phe19, Trp23, and Leu26). The molecular scaffolds which mimic the Phe19, Trp23, and Leu26 inhibit the binding of p53 to MDM2 [2]. Thus, prevent degradation of p53 and maintain its high level for induction of apoptosis in cancer cells. Earlier, diversity of small molecular inhibitors of p53-MDM2 interaction have been reported such nutlins (nutlin-3a), spirooxindoles (SAR405838), dihydroisoquinolinones (NVP-CGM097), and oxazoloisoindolinones (DIMP53-1) [3-6]. Most of inhibitors of p53-MDM2 interaction have multiples chiral centers so that their synthesis, as well as purification, is a tedious process. Earlier, we have successfully synthesized various dihydropyrimidinones using Lewis acid catalyzed multi-component synthesis $[7,8]$. Here, we reported a dihydropyrimidinone based novel potential lead (L11) for inhibition of p53-MDM2 interaction. The design of L11 was obtained after sequential screening of more than 500 dihydropyrimidinones by Lead IT program. The IC $_{50}$ values of L11 predicted in various pancreatic cell lines indicated its high potential as an anticancer agent. In addition, comparison of anticancer activity, toxicity profile and metabolism profile with known potential inhibitors of p53-MDM2 interaction (nutlin-3a and SAR405838) also added a further potential for biological activity.

\section{METHODS}

\section{Molecular docking study}

A database library of all the structures used in docking study was generated by Chem3D Ultra (v.10.0) as mol files after energy of each structure was minimized using MM2 method. The 3D structures of protein MDM2 along with cocrystallized ligands (pdb id: 1YCR, 4J3E, and 5TRF) were obtained from RCSB protein data bank. The preparation of receptor and docking of each database was done by Lead IT software using cocrystallized ligand as a reference. This provided and ranked all possible conformations of a single data based along with their Pose Views and docking scores. The binding affinity and ligand efficiency each conformation with docking score $<-10$ was done using "Hyde" program module. The isosteric replacement of functional groups in docked compounds was done using "ReCore" module. All the Lead IT modules were obtained for computational study part of project work with BiosolvelT (Germany).

\section{Prediction of anticancer activity}

The anticancer activity of each compound (including reference) was determined in comparison with most active anticancer agent and inactive anticancer agents in National Cancer Institute (NCI) database using "Cancer IN" server [9]. SMILE files of each compound were generated using Chem3D were used as input files for "Cancer IN." This provided hybrid score, tanimoto coefficient score, potency score (PS), mean $\log \mathrm{IG}_{50}$, and best hit with NSC ID. "CDRUG" server was used for further verification of anticancer activity profile of all compounds [10].

Prediction of IC

The $\mathrm{IC}_{50}$ value of each compound was determined using "DiPCell" server in five pancreatic cell lines, i.e., AsPC-1 (p53 null), BxPC-3 (p53-mutant), 
CAPAN-1 (p53-mutant), MIA-PaCa-2 (p53-mutant), and CAPAN-2 (p53wild type) [11]. SMILE files of each compound were generated using Chem3D which transformed into SDF input files for "DiPCell" with the help of "CACTUS SMILES Translator" server. For IC ${ }_{50}$ prediction default value selected as zero. The predicted $\mathrm{IC}_{50}$ values further transformed into a correlation plot of $\mathrm{IC}_{50}$ versus cell lines and $\log \mathrm{P}$ value of each compound was generated as a molecular descriptor.

\section{Prediction of toxicity}

The toxicity of each compound (including reference) was predicted for oral route administration using Prediction of Rodent Oral TOXicity (PROTOX) server. The chemical structure of each input compound for PROTOX was generated using inbuilt 2D chemical structure drawing tool. This provided $\mathrm{LD}_{50}(\mathrm{mg} / \mathrm{Kg})$, toxicity class, average similarity (\%), and predicted accuracy (\%) [12]

\section{Prediction of metabolism}

Metabolic profile of compounds was predicted by two different servers - "RS-Predictor" and "MetaPrint2D" [13,14]. The SDF or SMILE files used as input files in both servers were generated by Chem3D. "RS-Predictor" predict sites of metabolism as well as region selectivity by various CYPs. The "ALL (Metabolite 2010.2)" model, which allows metabolic site prediction in human, dog and rat models, for generation of metabolic data by "MetaPrint2D." While MetaPrint2D-react' server was used for determination of preferred metabolic reaction for site identified by "MetaPrint2D" [15].

\section{RESULTS AND DISCUSSION}

\section{Docking study of nutlin-3a, SAR405838 and L0-L11 in MDM2}

Dihydropyrimidinones are an important class of heterocyclic with diverse biological activities which can be synthesized using ethyl acetoacetate, aromatic aldehyde, and urea or thiourea in the presence of Lewis acid catalyst. They can be transformed into structurally diverse molecular scaffolds by substitution with different functional groups. In search of p53-MDM2 interaction inhibitors, we designed dihydropyrimidinone based inhibitors using Lead IT software package. In an early screening study of more than 170 dihydropyrimidinones, compound L0 was found to be most active and starting skeleton for further optimization. It showed comparable results with known p53MDM2 interaction inhibitors nutlin-3a and SAR405838 [16]. The data from comparative study were used for further optimization of L0 by generating more than 400 primary daughter ligands with structural modifications. Further, isosteric modification by "ReCore" and docking provided 11 most active ligands L1-L11 (Table 1) as compared to L0. Screening of R/S isomers of ligands L1-L11 provided (S)-L11 as most potent inhibitor of p53-MDM2 interaction. (S)-L11 showed the MDM2 binding pattern similar to that of reference, nutlin-3a and SAR405838 [17,18]. It successfully mimics three crucial residues (Phe19, trp23, and Leu26) of p53 in binding cavity on MDM2 (Fig. 1a) [19]. The chemical structures for SAR405838 and L11 are represented in (Fig. 1b). The Bromoindole ring and 5-bromo-2-methylbenzyl moiety oriented in Trp23 (p53) and Leu26 (p53) binding cavities, respectively. Later also participated in $\pi-\pi$ stacking interaction with His 96 of MDM2 which is essential for stable binding [20]. While $\mathrm{N}-\mathrm{H}$ of indole ring participated in H-bond formation with Leu64 of MDM2. The 4-bromo2-methoxybenzoyl moiety hydrophobically interacts with Met62, Tyr67, and Val93 of MDM2 in Phe19 (p53) binding cavity. The 3-ethyl5 -methoxybenzyl stabilizes capping of binding cavity by hydrophobic interactions at surface with Ile61 and Lys94 of MDM2. The 2-methoxy substituent showed additional interaction as an H-bond with side chain of Lys94 of MDM2 and stabilizes orientation of phenyl ring similar to the previous study [21]. The carbonyl group of dihydropyrimidinone ring forms H-bond with imidazole ring of His 96 and is responsible for better fitting of the molecule (Fig. 1c). As indicated by docking data, the docking scores of L3-L11 found to be in range of 21-25 close to docking score 25 of SAR405838 and better than nutlin-3a. MDM2 binding

Table 1: Docking data ligands after optimization

\begin{tabular}{lllll}
\hline Ligands & $\begin{array}{l}\text { Docking } \\
\text { score }\end{array}$ & $\begin{array}{l}\text { Binding } \\
\text { affinity } \mathbf{(} \boldsymbol{\Delta G}) \\
\text { KJ/mol }\end{array}$ & $\begin{array}{l}\text { Ligand } \\
\text { efficiency (LE) } \\
\text { KCal/mol }\end{array}$ & LogP \\
\hline Nutlin-3a & -16.7852 & -37 & 0.22 & 5.436 \\
SAR405838 & -25.4693 & -78 & 0.49 & 5.479 \\
L0 & -17.1810 & -37 & 0.27 & 4.788 \\
L1 & -14.6126 & -47 & 0.28 & 5.362 \\
L2 & -14.2268 & -42 & 0.23 & 7.047 \\
L3 & -22.1801 & -55 & 0.32 & 7.548 \\
L4 & -23.8355 & -54 & 0.32 & 9.004 \\
L5 & -23.6802 & -54 & 0.30 & 7.627 \\
L6 & -25.0627 & -62 & 0.33 & 8.710 \\
L7 & -22.6016 & -65 & 0.34 & 8.254 \\
L8 & -22.5109 & -65 & 0.32 & 9.353 \\
L9 & -23.0506 & -67 & 0.33 & 9.484 \\
L10 & -22.3624 & -70 & 0.34 & 9.899 \\
L11 & -21.7687 & -78 & 0.37 & 10.316 \\
\hline
\end{tabular}

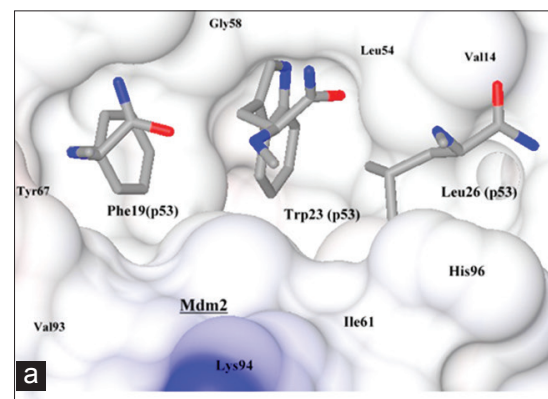<smiles>CC(C)(C)C[C@H]1N[C@H](C(=O)NC2CCC(O)CC2)[C@H](c2cccc(Cl)c2F)[C@@]12C(=O)Nc1cc(Cl)ccc12</smiles>

b SAR405838<smiles>CCc1ccc(Br)cc1CN1C(=O)N(Cc2cc(C[AlH])cc(OC)c2)C(C)=C(C(=O)c2ccc(Br)cc2OC)[C@H]1c1c[nH]c2cc(Br)ccc12</smiles>

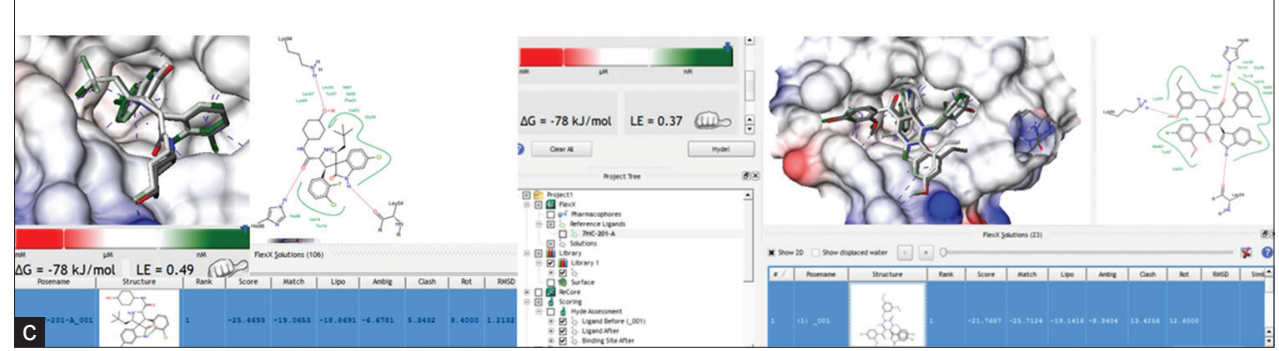

Fig. 1: Ligand L11 binds in p53-binding cavity on MDM2. (a) Crucial AA residues of p53, (b) structures of SAR405838 and L11, (c) docking and Hyde assessment of SAR405838 and L11 
affinity values (37-78 Kcal $/ \mathrm{mol}$ ) of all ligands were also found to better than that of nutlin-3a (37 Kcal/mol). Surprisingly, MDM2 binding affinity of L11 (78 Kcal/mol) found to be equal to that of SAR405838 and 2-fold greater than nutlin-3a (Table 1). These data are indicating that L11 as a potential lead from dihydropyrimidinones for inhibition of p53-MDM2 interaction.

\section{Anticancer activity profile prediction of nutlin-3a, SAR405838 and} LO-L11

Anticancer activity prediction was carried out as potency score (PS) using "Cancer IN" server (http://crdd.osdd.net/oscadd/cancerin/algo. php) as reported earlier [9].

Potency score was computed on the basis following equation:

$\mathrm{PS}=\operatorname{Max}\left(\mathrm{H}^{\mathrm{a}} \mathrm{T}_{\mathrm{s} 1}, \mathrm{H}^{\mathrm{a}} \mathrm{T}_{\mathrm{s} 0}\right)-\operatorname{Max}\left(\mathrm{H}^{\mathrm{n}} \mathrm{T}_{\mathrm{s} 1}, \mathrm{H}^{\mathrm{n}} \mathrm{T}_{\mathrm{s} 0}\right)$

Where Max is maximum score, $\mathrm{H}^{\mathrm{a}} \mathrm{T}$ indicates Tanimoto or Jaccard similarity score, $\mathrm{H}^{\mathrm{a}} \mathrm{T}_{\mathrm{s} 0}$ is highest similarity score between the query and most similar anticancer agents, $\mathrm{H}^{\mathrm{n}} \mathrm{T}_{\mathrm{si}}$ and $\mathrm{H}^{\mathrm{n}} \mathrm{T}_{\mathrm{s} 0}$ indicates similarity scores between the query and most similar nonanticancer agents [9,22]. The potency score values vary from -1 to +1 . The potency score provides the distance of query molecule with active and inactive anticancer inhibitors. Thus, a molecule having high potency score is more similar to active anticancer inhibitors as compare to inactive anticancer inhibitors [9]. In anticancer activity study compound, L9-L11 showed highest PS values (0.22) among the series as well as reference compounds (0.15-0.16). These data also indicated that L11 is approximately 1.5 times more potent than reference anticancer agents, nutlin-3a and SAR405838. The hybrid score was calculated to measure the similarity between the query and the active anticancer compounds. Among in the series, a higher value of hybrid score for L0 (0.4065) indicted its high similarity with NCI hit (729779). However, compound L9-L11 also showed hybrid score (0.338) better than reference compounds (Table 2).

For further verification of anticancer activity, all the compounds were screened using "CDRUG" server (http://bsb.kiz.ac.cn/CDRUG/) [10]. CDRUG uses a novel molecular description method, known as relative frequency-weighted fingerprint, to implement the molecular fingerprints and then uses a hybrid score for measurement of compound similarity. The maximum hybrid score value is equal to 1.0. Finally, hybrid score used to calculate a confidence level ( $p$ value) which predicted whether the test compounds (L0-L11) have or do not have the anticancer activity [10]. Among the series compound L11 showed confidence level $(\mathrm{p}=0.8153)$ approximate equal to that of SAR405838 $(\mathrm{p}=0.8210)$ and greater than that of nutlin-3a $(\mathrm{p}=0.7072)$ (Table 3$)$. These data also indicted that L11 has the highest potential as an anticancer agent in the series.

\section{IC $_{50}$ values prediction of nutlin-3a, SAR405838 and L0-L11}

The compound L0-L11 was evaluated for their potency by prediction of IC ${ }_{50}$ value and compared with reference ligands. For prediction, the pancreatic cell lines AsPC-1 (p53 null), BxPC-3 (p53-mutant), CAPAN-1 (p53-mutant), MIA-PaCa-2 (p53-mutant), and CAPAN-2 (p53-wild type) were selected on the basis on previous biological evaluation studies for p53-MDM2 inhibitors [23,24]. DiPCell server reported earlier and known for prediction of anticancer activity ( $\mathrm{IC}_{50}$ values) precisely for various types of pancreatic cell lines (http://crdd.osdd.net/raghava/ dipcell/) [11]. The DiPCell predicted $\mathrm{IC}_{50}$ values which ranging from $-7 \mu \mathrm{M}$ (most sensitive) to $+7 \mu \mathrm{M}$ (most resistant). Thus, anticancer activity of compounds L0-L11 predicted and compared with standards nutlin-3a and SAR405838. The data indicated that compound L11 shows potential anticancer activity as compared to other derivatives of the series and well as standards. Compound L3, L7, and L9 were found to be active against CAPAN- 1 cell lines with IC $_{50}$ values of -0.164 , -0.687 , and $-0.687 \mu \mathrm{M}$, respectively. While compound L5 and L6 found to be active against BxPC- 3 cell line with $\mathrm{IC}_{50}$ values of -0.429 and $-1.360 \mu \mathrm{M}$, respectively. Among the series, only compound L11 was active against MIA-PaCa- 2 cell line with the $\mathrm{IC}_{50}$ value of $-0.323 \mu \mathrm{M}$
Table 2: Comparative anticancer activity profile of compound LO-L11, nutlin-3a, and SAR405838

\begin{tabular}{llllll}
\hline Compounds & $\begin{array}{l}\text { Hybrid } \\
\text { score }\end{array}$ & TC & PS & Mean log IG 50 & $\begin{array}{l}\text { Best Hit } \\
\text { NSC ID }\end{array}$ \\
\hline Nutlin-3a & 0.2665 & 0.33 & 0.16 & -5.027 & 133118 \\
SAR405838 & 0.2205 & 0.36 & 0.15 & -7.544 & 7534 \\
L0 & 0.4065 & 0.81 & 0.22 & -4.963 & 729779 \\
L1 & 0.235 & 0.32 & 0.13 & -7.645 & 135036 \\
L2 & 0.308 & 0.35 & 0.16 & -6.216 & 748671 \\
L3 & 0.3055 & 0.52 & 0.19 & -5.549 & 7571 \\
L4 & 0.309 & 0.51 & 0.19 & -5.492 & 87206 \\
L5 & 0.3165 & 0.46 & 0.20 & -7.528 & 7532 \\
L6 & 0.3165 & 0.46 & 0.20 & -7.528 & 7532 \\
L7 & 0.31 & 0.47 & 0.21 & -5.682 & 135037 \\
L8 & 0.31 & 0.47 & 0.21 & -5.682 & 135037 \\
L9 & 0.338 & 0.48 & 0.22 & -5.254 & 748715 \\
L10 & 0.338 & 0.48 & 0.22 & -5.254 & 748715 \\
L11 & 0.338 & 0.48 & 0.22 & -5.254 & 748715 \\
\hline
\end{tabular}

TC: Tanimoto coefficient score, PS: Potency score

Table 3: CDRUG predicted data for L0-L11, nutlin-3a, and SAR405838

\begin{tabular}{llll}
\hline Compounds & p-value & ${\text { Mean } \mathbf{l o g} \mathbf{I G}_{\mathbf{5 0}}}$ & Hybrid score \\
\hline Nutlin-3a & 0.7072 & -5.058 & 0.081 \\
SAR405838 & 0.8210 & -5.130 & 0.062 \\
L0 & 0.0722 & -5.159 & 0.334 \\
L1 & 0.4003 & -5.007 & 0.140 \\
L2 & 0.5040 & -5.159 & 0.117 \\
L3 & 0.6119 & -5.007 & 0.097 \\
L4 & 0.6293 & -5.232 & 0.094 \\
L5 & 0.6708 & -5.712 & 0.087 \\
L6 & 0.7498 & -5.007 & 0.074 \\
L7 & 0.7619 & -5.712 & 0.072 \\
L8 & 0.7619 & -5.007 & 0.072 \\
L9 & 0.7619 & -5.007 & 0.072 \\
L10 & 0.7679 & -5.007 & 0.071 \\
L11 & 0.8153 & -5.007 & 0.063 \\
\hline
\end{tabular}

Table 4: Predicted $\mathrm{IC}_{50}$ values $(\mu \mathrm{M})$ of ligands L0-L11 for different types of cancer cell line

\begin{tabular}{llllll}
\hline $\begin{array}{l}\text { Cell lines } \\
\text { Ligands }\end{array}$ & AsPC-1 & BxPC-3 & CAPAN-1 & MIA-PaCa-2 & CAPAN-2 \\
\hline Nutlin-3a & 2.748 & -1.122 & -1.886 & -2.004 & 0.774 \\
SAR405838 & 0.685 & -1.548 & -0.185 & -1.006 & 2.635 \\
L0 & 2.803 & 1.668 & 1.175 & 0.184 & 0.932 \\
L1 & 3.331 & 0.552 & 2.344 & 3.638 & 1.176 \\
L2 & 3.698 & 2.006 & 1.787 & 3.981 & 2.269 \\
L3 & 2.405 & 0.745 & -0.164 & 2.788 & 1.832 \\
L4 & 5.251 & 0.702 & 1.250 & 3.222 & 1.873 \\
L5 & 3.403 & -0.429 & 1.579 & 1.160 & 1.405 \\
L6 & 3.459 & -1.360 & 0.592 & 0.167 & 1.167 \\
L7 & 4.300 & 2.165 & -0.687 & 0.645 & 1.029 \\
L8 & 4.220 & 0.029 & 0.853 & 0.485 & 1.136 \\
L9 & 4.300 & 1.062 & -0.687 & 0.645 & 1.029 \\
L10 & 4.220 & 0.029 & 0.853 & 0.485 & 1.136 \\
L11 & 3.932 & -0.408 & -1.790 & -0.323 & 1.991 \\
\hline
\end{tabular}

(Table 4). Along with nutlin-3a and SAR405838, compound L11shows selectivity against BxPC-3, CAPAN-1 MIA-PaCa-2 cell lines. Moreover, L11 indicated higher potency against CAPAN-1 $\left(\mathrm{IC}_{50}=-1.79 \mu \mathrm{M}\right)$ than SAR405838 $\left(\mathrm{IC}_{50}=-0.185 \mu \mathrm{M}\right)$ (Fig. 2).

$\mu$ Toxicity profile prediction of nutlin-3a, SAR405838 and L0-L11 Finally, the toxicity profiles of all the compounds were prediction using "PROTOX" server (http://tox.charite.de/tox/) [12]. "PROTOX" predicts oral toxicities of small molecules in rodents on the basis 
of chemical similarities between compounds with known toxic effects and the presence of toxic fragments. Toxic doses are often given as dose at which $50 \%$ of test subjects die on exposure to a compound $\left(\mathrm{LD}_{50}\right.$ values $)$ in $\mathrm{mg} / \mathrm{kg}$ body weight. It also classifies the test compounds in I-VI classes as per standard format based on $\mathrm{LD}_{50}$ values [12]. The $\mathrm{LD}_{50}$ of nutlin-3a predicted as $400 \mathrm{mg} / \mathrm{kg}$. As reported

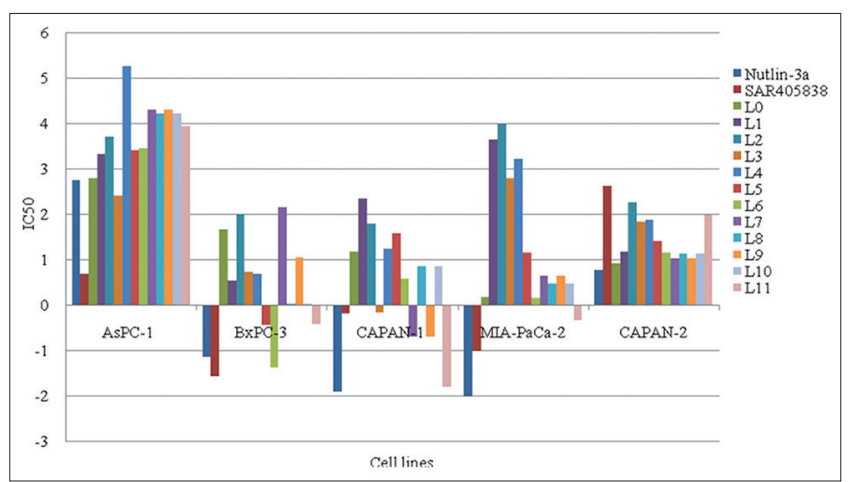

Fig. 2: Predicted anticancer activity of nutlin-3a, SAR405838, and L0-L11
Table 5: Predicted toxicity profiles of nutlin-3a, SAR405838, and compound L0-L11

\begin{tabular}{lllll}
\hline Compounds & LD $_{\mathbf{5 0}}(\mathbf{m g} / \mathbf{K g})$ & $\begin{array}{l}\text { Toxicity } \\
\text { class* }\end{array}$ & $\begin{array}{l}\text { Average } \\
\text { similarity } \\
\text { (\%) }\end{array}$ & $\begin{array}{l}\text { Predicted } \\
\text { accuracy } \\
\text { (\%) }\end{array}$ \\
\hline Nutlin-3a & 400 & IV & 43.26 & 54.26 \\
SAR405838 & 36 & II & 45.38 & 54.26 \\
L0 & 1500 & IV & 42.53 & 54.26 \\
L1 & 1000 & IV & 43.32 & 54.26 \\
L2 & 1000 & IV & 42.80 & 54.26 \\
L3 & 500 & IV & 44.53 & 54.26 \\
L4 & 200 & IV & 39.26 & 23.00 \\
L5 & 900 & IV & 44.20 & 54.26 \\
L6 & 500 & IV & 43.68 & 54.26 \\
L7 & 500 & IV & 42.41 & 54.26 \\
L8 & 500 & IV & 42.27 & 54.26 \\
L9 & 500 & IV & 41.75 & 54.26 \\
L10 & 500 & IV & 41.93 & 54.26 \\
L11 & 1644 & IV & 42.12 & 54.26 \\
\hline
\end{tabular}

*Class I: Fatal if swallowed (LD50 $\leq 5 \mathrm{mg} / \mathrm{kg}$ ), Class II:

Fatal if swallowed $(5<\mathrm{LD} 50 \leq 50 \mathrm{mg} / \mathrm{kg})$, Class III: Toxic if swallowed $(50<\mathrm{LD} 50 \leq 300 \mathrm{mg} / \mathrm{kg})$, Class IV: Harmful if swallowed $(300<\mathrm{LD} 50 \leq 2000 \mathrm{mg} / \mathrm{kg})$, Class V: May be harmful if swallowed $(2000<\mathrm{LD} 50 \leq 5000 \mathrm{mg} / \mathrm{kg})$, Class VI: Non-toxic (LD50>5000 mg/kg)

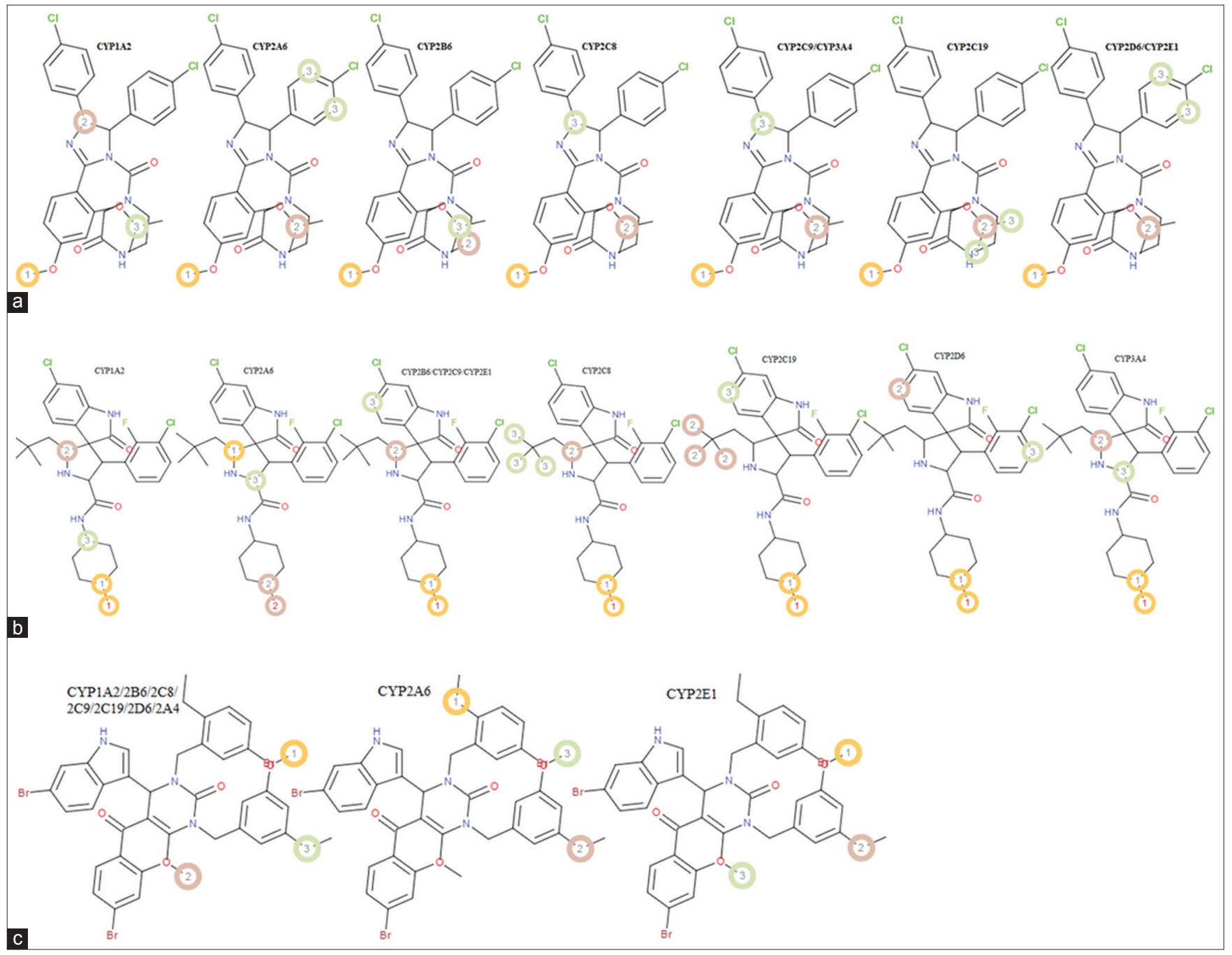

Fig. 3: Visual representation of metabolic sites for different cytochromes P450 s predicted by "RS-Predictor." (a) Preferred sites of metabolism of nutlin-3a, (b) preferred sites of metabolism of SAR405838, and (c) preferred sites of metabolism of L11. Numbers in yellow, red, and green circles indicated primary, secondary, and tertiary sites of metabolism in the compounds 


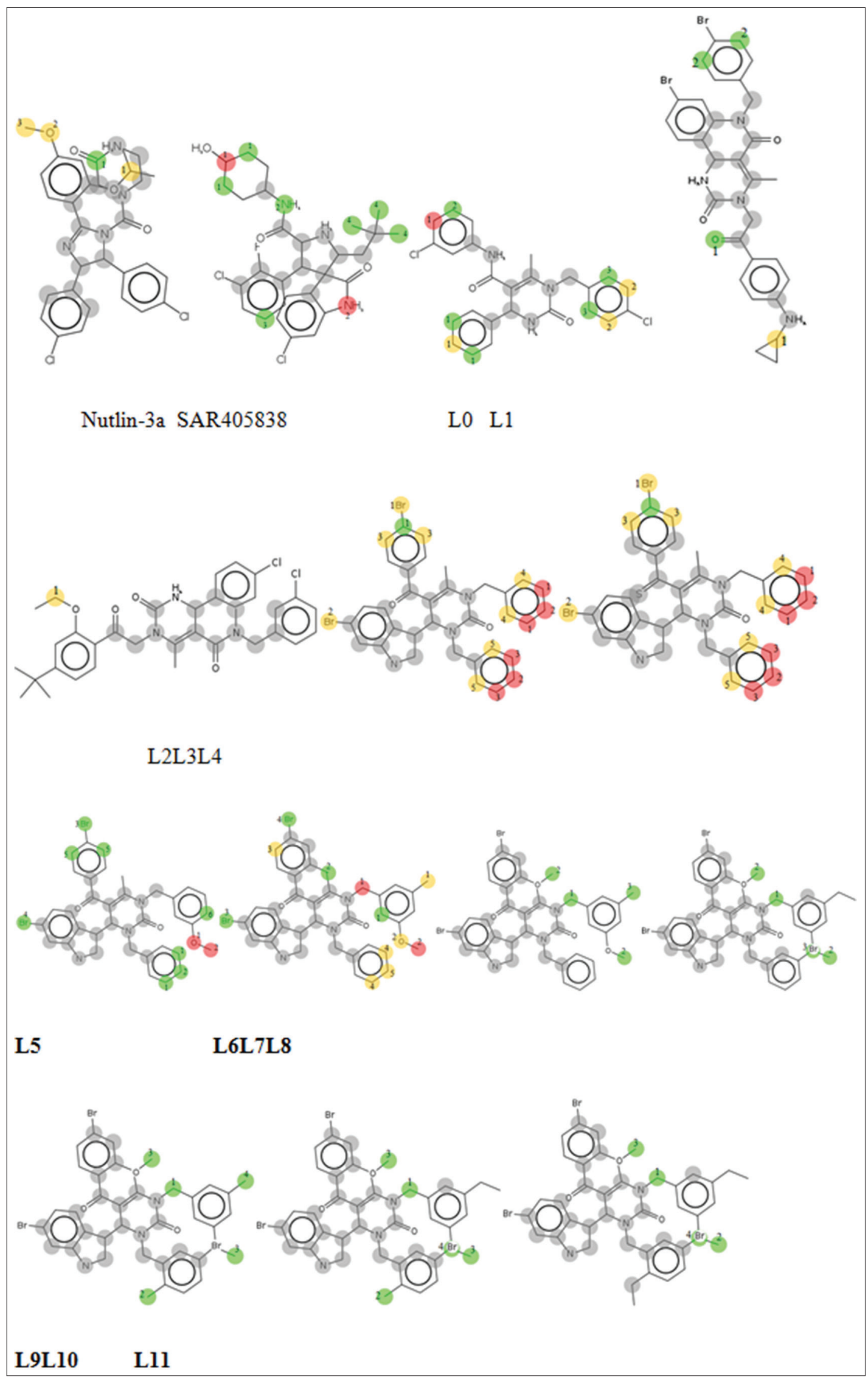

Fig. 4: Sites of metabolism of nutlin-3a, SAR405838 and L0-L11 by predicted by "MetaPrint2D." * $0.66 \leq \mathrm{NOR} \leq 1.00, \bigcirc 0.33 \leq \mathrm{NOR}<0.66$, $0.15 \leq \mathrm{NOR}<0.33$, little/no data, positions without circle $0.00 \leq \mathrm{NOR}<0.15$. The color highlighting an atom indicates its normalized occurrence ratio (NOR). A high NOR indicates a more frequently reported site of metabolism in the metabolite database. The "ALL (Metabolite 2010.2)" model was used for prediction of metabolism site 
Table 6: NOR for metabolism of nutlin-3a, SAR405838, and L0-L11

\begin{tabular}{|c|c|c|c|c|c|c|c|}
\hline \multirow[t]{2}{*}{ Compounds } & \multicolumn{2}{|c|}{ Site-A NOR } & \multicolumn{2}{|c|}{ Site-B NOR } & \multicolumn{2}{|c|}{ Site-C NOR } & \multirow[t]{2}{*}{ Metabolic reactions } \\
\hline & 1 & 2 & 1 & 2 & 1 & 2 & \\
\hline Nutlin-3a & - & - & 0.53 & 0.52 & 0.25 & - & Demethylation, hydroxylation \\
\hline SAR405838 & 0.78 & 0.74 & - & - & 0.29 & 0.17 & $\mathrm{~N}$-dealkylation, methylation, sulfation, glucuronidation \\
\hline L0 & 1.00 & - & 0.60 & 0.57 & 0.31 & 0.21 & Hydroxylation, glucuronidation \\
\hline L1 & - & - & 0.41 & - & 0.30 & 0.18 & Reduction, hydroxylation, $N$-dealkylation, oxidative deamination \\
\hline L2 & - & - & 0.33 & - & - & - & Dealkylation, oxidation, hydroxylation \\
\hline L3 & 1.00 & 0.83 & 0.65 & 0.55 & 0.27 & - & Oxidation, dehalogenation, hydroxylation \\
\hline L4 & 1.00 & 0.83 & 0.65 & 0.55 & 0.27 & - & Oxidation, dehalogenation, hydroxylation \\
\hline L5 & 1.00 & 0.67 & - & - & 0.32 & 0.32 & Dealkylation, oxidation, hydroxylation, dehalogenation \\
\hline L6 & 1.00 & 0.70 & 0.64 & 0.59 & 0.32 & 0.26 & Dealkylation, oxidation, hydroxylation, dehalogenation \\
\hline L7 & - & - & - & - & 0.25 & 0.17 & O-dealkylation, $\mathrm{N}$-dealkylation, hydroxylation \\
\hline L8 & - & - & - & - & 0.25 & 0.17 & O-dealkylation, $\mathrm{N}$-dealkylation, hydroxylation \\
\hline L9 & - & - & - & - & 0.25 & 0.21 & O-dealkylation, $\mathrm{N}$-dealkylation, hydroxylation \\
\hline L10 & - & - & - & - & 0.25 & 0.21 & O-dealkylation, $\mathrm{N}$-dealkylation, hydroxylation \\
\hline L11 & - & - & - & - & 0.25 & 0.17 & O-dealkylation, N-dealkylation, hydroxylation \\
\hline
\end{tabular}

NOR: Normalized occurrence ratio

earlier, nutlin-3a found to induce maximum cell death at $400 \mathrm{mg} / \mathrm{kg}$ single dose for 24 hrs break in RKO and SJSA-1 cell lines than split doses for 12 and $6 \mathrm{hr}$ breaks [25]. All the test compounds (L0-L11) found to be harmful if swallowed similar to reference nutin-3a. As $\mathrm{LD}_{50}$ data indicated that $\mathrm{L} 0\left(\mathrm{LD}_{50}=1500 \mathrm{mg} / \mathrm{kg}\right)$ and $\mathrm{L} 11$ are less harmful $\left(\mathrm{LD}_{50}=1644 \mathrm{mg} / \mathrm{kg}\right)$ than other members of the series and thus it is suitable for oral route of administration in small doses. However, data for reference SAR4059838 indicated its fatality $\left(\mathrm{LD}_{50}=36 \mathrm{mg} / \mathrm{kg}\right)$ on administration through oral route (Table 5).

Metabolism profile prediction of nutlin-3a, SAR405838 and L0-L11 Cytochrome P450 enzymes (CYPs) are involved in Phase-I metabolism of drugs. However, most of therapeutic agents are metabolized by eight isoforms (CYP1A2, CYP2A6, CYP2B6, CYP2C9, CYP2C19, CYP2D6, CYP3E1, and CYP3A4) [26,27]. Among all isoforms of CYPs, CYP3A4 metabolizes majority of clinically used drugs. CYPs catalyzes variety of biotransformations such as $\mathrm{S}$ - and $\mathrm{N}$-oxidation, aromatic and aliphatic oxidation, $\mathrm{N}$ - and O-dealkylation, sulfoxide/ sulfone formation, oxidative deamination, dehalogenation, and desulfuration [28]. At the time of initial discovery, the metabolic fates of potential therapeutic lead compounds are often unknown. A prior knowledge of their metabolic fates could have important ramifications in the cost and speed of the drug development process. Thus, we predicted the pharmacokinetic profile of compound L11 and references using "RS-Predictor" server (http://reccr.chem.rpi. edu/Software/RS-WebPredictor/) [13]. "RS-Predictor" has been used successfully for prediction of metabolic sites and CYP regioselectivity in several drugs such as alpha dihydroergocryptine, bromocriptine, bupropion, diclofenac, tertranor, pradefovir, nitropyrene, arachidonic acid, phenprocoumon, pinacidil, etoposide, docetaxel, tentoxin, and chlorpromazine. [13]. Earlier studies have indicated that nutlin-3a and SAR405838 metabolized by liver microsomes [4,29]. However, detail of their metabolic pathways and metabolites are undisclosed. Hence, we selected both reference compounds for identification of their preferred site, microsomal enzymes and metabolic reactions. The obtained data on metabolism were further compared with metabolism data of L11. Predicted result showed that nutlin-3a has primary and secondary or tertiary sites of metabolism as methoxy and isopropoxy substituent, respectively, for various CYPs. Thus, it indicated that nutlin-3a is metabolized by 0 -dealkylation and hydroxylation. Result for SAR405838 showed that it preferably metabolized by dehydroxylation of 4-OH group in cyclohexyl side chain. While preferred sites of metabolism of L11 found to be methoxy substituent in 2-ethyl-5-methoxybenzyl side chain through 0-demethylation (Fig. 3).

Furthermore, metabolism profile of compound L0-L11 and references also predicted using "MetaPrint2D" and "MetaPrint2D-React" servers of
Cambridge University $[14,15]$. It was done so that any wrong prediction can be checked. For prediction of metabolism site, ALL model (human, dog, mice, and rate) and parameter setting were selected as default. As data indicated that preferred sites of metabolism of nultin-3a (NOR=0.53) and SAR405838 (NOR=0.78) by CYPs are similar to that which predicted by "RS-Predictor." As expected, L11 showed methoxy of 2-ethyl-5-methoxybenzyl side chain as a site of metabolic in top two ranked sites (NOR $=0.25$ and 0.17) (Fig. 4 and Table 6).

\section{CONCLUSION}

Mimicking three residues (Phe19, Trp23, and Leu26) of p53 has been recognized as a valid strategy for the development of p53-MDM2 interaction inhibitor based novel anticancer agents. Dihydropyrimidinone based scaffolds provided the opportunity to transform them into a lead for inhibition of p53-MDM2 interaction. During screening of $>500$ dihydropyrimidinones through a docking study, compound L11 was found to mimic the three residues of p53 efficiently and effectively. Compound L11 also proved its potential as an anticancer agent based on p53-MDM2 interaction inhibition as indicated by its anticancer activity, low $\mathrm{IC}_{50}$ values in different cell lines, toxicity profile, and metabolism profile. When compared with nutlin-3a and SAR405838, compound L11 showed comparable or better results. Thus, computational study indicated its success as a p53-MDM2 interaction inhibitor and anticancer agent. However, there is need to study binding and activity of L11 in living system.

\section{ACKNOWLEDGMENT}

Authors are thankful to Senior Dean, School of Pharmaceutical Sciences, Lovely Professional University (India) and BiosolvelT (Germany), for providing work facilities.

\section{REFERENCES}

1. Nayak SK, Panesar PS, Kumar H. Non-genotoxic p53-activators and their significance as antitumor therapy of future. Curr Med Chem 2011;18(7):1038-49.

2. Böttger $A$, Böttger V, Garcia-Echeverria $C$, Chène $P$, Hochkeppel HK, Sampson W, et al. Molecular characterization of the hdm2-p53 interaction. J Mol Biol 1997;269(5):744-56.

3. Secchiero P, di Iasio MG, Gonelli A, Zauli G. The MDM2 inhibitor nutlins as an innovative therapeutic tool for the treatment of haematological malignancies. Curr Pharm Des 2008;14(21):2100-10.

4. Zhao Y, Yu S, Sun W, Liu L, Lu J, McEachern D, et al. A potent small-molecule inhibitor of the MDM2-p53 interaction (MI-888) achieved complete and durable tumor regression in mice. J Med Chem 2013;56(13):5553-61.

5. Holzer P, Masuya K, Furet P, Kallen J, Valat-Stachyra T, Ferretti S, et al. Discovery of a dihydroisoquinolinone derivative (NVP-CGM097): A highly potent and selective MDM2 inhibitor undergoing phase 1 
clinical trials in p53wt tumors. J Med Chem 2015;58(16):6348-58.

6. Soares J, Espadinha M, Raimundo L, Ramos H, Gomes AS, Gomes S, et al. DIMP53-1: A novel small-molecule dual inhibitor of p53MDM2/X interactions with multifunctional p53-dependent anticancer properties. Mol Oncol 2017;11(6):612-27.

7. Puri S, Kaur B, Parmar A, Kumar H. Ultrasound promoted $\mathrm{Cu}(\mathrm{Clo} 4) 2$ catalyzed rapid synthesis of substituted1, 2, 3, 4-tetrahydropyrimidine2-ones \& hantzsch 1, 4-dihydropyridines in dry media. Heterocycl Comm 2009;15(1):51-5.

8. Kaur M. Study of Perchlorates as Catalysts for Construction of Organic Molecular Frameworks. Master's Dissertation, Lovely Professional University; 2014.

9. Singh H, Kumar R, Singh S, Chaudhary K, Gautam A, Raghava GP. Prediction of anticancer molecules using hybrid model developed on molecules screened against NCI-60 cancer cell lines. BMC Cancer 2016;16:77.

10. Li GH, Huang JF. CDRUG: A web server for predicting anticancer activity of chemical compounds. Bioinformatics 2012;28(24):3334-5.

11. Kumar R, Chaudhary K, Singla D, Gautam A, Raghava GP. Designing of promiscuous inhibitors against pancreatic cancer cell lines. Sci Rep 2014;4:4668.

12. Drwal MN, Banerjee P, Dunkel M, Wettig MR, Preissner R. ProTox: A web server for the in silico prediction of rodent oral toxicity. Nucleic Acids Res 2014;42:W53-8.

13. Zaretzki J, Bergeron C, Rydberg P, Huang TW, Bennett KP, Breneman CM. RS-predictor: A new tool for predicting sites of cytochrome P450-mediated metabolism applied to CYP 3A4. J Chem Inf Model 2011;51(7):1667-89.

14. Available from: http://www.www-metaprint2d.ch.cam.ac.uk/ metaprint2d. [Last cited on 2017 Mar 27].

15. Available from: http://www.www-metaprint2d.ch.cam.ac.uk/ metaprint2d-react. [Last cited on 2017 Mar 27].

16. Burgess A, Chia KM, Haupt S, Thomas D, Haupt Y, Lim E. Clinical overview of MDM2/X-targeted therapies. Front Oncol 2016;6:7.

17. Wang S, Zhao Y, Bernard D, Aguilar A, Kumar S. Targeting the MDM2-p53 protein-protein interaction for new cancer therapeutics. Top Med Chem 2012;8:57-80.

18. Wang S, Sun W, Zhao Y, McEachern D, Meaux I, Barrière C, et al. SAR405838: An optimized inhibitor of MDM2-p53 interaction that induces complete and durable tumor regression. Cancer Res 2014;74(20):5855-65.

19. Liu SX, Geng YZ, Yan SW. Structural effects and competition mechanisms targeting the interactions between p53 and MDM2 for cancer therapy. Front Phys 2017;12:128908.

20. Ribeiro CJ, Rodrigues CM, Moreira R, Santos MM. Chemical variations on the p53 reactivation theme. Pharmaceuticals (Basel) 2016;9(2). pii:E25.

21. Danelius E, Pettersson M, Bred M, Min J, Waddell MB, Guy RK, et al. Flexibility is important for inhibition of the MDM2/p53 protein-protein interaction by cyclic ß-hairpins. Org Biomol Chem 2016;14(44):10386-93

22. Butina D. Unsupervised data base clustering based on daylight's fingerprint and animator similarity: A fast and automated way to cluster small and large data sets. J Chem Inf Comput Sci 1999;39(4):747-50.

23. Wang W, Qin JJ, Voruganti S, Wang MH, Sharma H, Patil S, et al. Identification of a new class of MDM2 inhibitor that inhibits growth of orthotopic pancreatic tumors in mice. Gastroenterology 2014;147(4):893-902.e2.

24. Azmi AS, Aboukameel A, Banerjee S, Wang Z, Mohammad M, Wu J, et al. MDM2 inhibitor MI-319 in combination with cisplatin is an effective treatment for pancreatic cancer independent of p53 function. Eur J Cancer 2010;46(6):1122-31.

25. Puszynski K, Gandolfi A, d'Onofrio A. The pharmacodynamics of the p53-Mdm2 targeting drug nutlin: The role of gene-switching noise. PLoS Comput Biol 2014;10(12):e1003991.

26. Zanger UM, Schwab M. Cytochrome P450 enzymes in drug metabolism: Regulation of gene expression, enzyme activities, and impact of genetic variation. Pharmacol Ther 2013;138(1):103-41.

27. Tan CY, Saw TY, Fong CW, Ho HK. Comparative hepatoprotective effects of tocotrienol analogs against drug-induced liver injury. Redox Biol 2015;4:308-20.

28. Lamb DC, Waterman MR, Kelly SL, Guengerich FP. Cytochromes P450 and drug discovery. Curr Opin Biotechnol 2007;18(6):504-12.

29. Zhang F, Tagen M, Throm S, Mallari J, Miller L, Guy RK, et al. Wholebody physiologically based pharmacokinetic model for nutlin-3a in mice after intravenous and oral administration. Drug Metab Dispos 2011;39(1):15-21 